\title{
CORROSION BEHAVIOUR OF ALUMINIUM 6061 NICKEL COATED CENOSPHERE COMPOSITE
}

\author{
Arvind R S ${ }^{1}$, Prasanna Ram M ${ }^{1}$, *Prashanth $T^{1}$, Jaimon Dennis Quadros ${ }^{2}$
}

${ }^{1}$ Department of Mechanical Engineering, Global Academy of Technology, Bangalore, Karnataka-560098, India ${ }^{2}$ Department of Mechanical Engineering, Birla Institute of Technology, RAK- 41222, United Arab Emirates https://doi.org/10.37255/jme.v15i3pp54-59

\begin{abstract}
Cenosphere fly ash is one of the most inexpensive and low-density material which is abundantly available as a solid waste by-product of coal combustion in thermal power plants. Aluminium metal matrix composites with Nickel coated cenospheres as the reinforcement is prepared by stir casting route. The composites are prepared with varying percentages of cenospheres in the percentage of $2-10 \%$ by weight of the composite. Immersion corrosion tests are conducted on the composites in three different medium and for three different time durations. It is evident from the test results as well as the microstructure images that the weight loss of samples with $8 \%$ Nickel coated cenospheres has shown least corrosion or the highest corrosion resistance when compared to the counterparts.
\end{abstract}

Keywords: Aluminium 6061 composites, fly ash cenospheres, Nickel coated cenospheres, corrosion resistance, immersion corrosion.

\section{Introduction}

Aluminium and its alloys are of high specific strength and low density. Therefore, they have a wide range of application in the automotive and aerospace industries [1]. Metal matrix composites (MMCs)are gaining widespread popularity in several technological fields owing to its improved mechanical properties when compared with conventional metals/alloys [2]. The reinforcements are generally used to improve the base metal properties such as strength, stiffness, conductivity, etc. [3]

India is the second-largest producer of coal in the world, generates copious amounts of fly ash. Since fly ash is a very light particle, it needs to be stored under an artificial storage pond; therefore, the demand to determine the usage of fly ash in metal matrix composites is increasing.

Though considered as a waste, cenosphere is a useful by-product to be used to produce newer materials cheaply which otherwise poses significant environmental and disposable problems. Fly-ash cenospheres have unique properties such as low density, non-toxic, non-metallic hollow microparticles and are light in weight. The particle size of cenospheres varies from 1 to $20 \mu \mathrm{m}$ and their mean particle diameter in the order of $8 \mu \mathrm{m}$. Cenosphere has a size range from 1 to 500 microns with an average compressive strength up to 7000 psi. Their colour ranges from white to dark grey. [4]

*Corresponding Author - E-mail: prashanth.t@gat.ac.in
Cenosphere reinforced aluminium enhances the chemical stability of aluminium-cenosphere composites during synthesis and reheating [5]. The weight loss of the MMC material followed descending order as percent filler increased till $6 \%$ cenosphere, further increase of cenosphere resulted with an increase in weight loss for both heat-treated and untreated composites [6]. Cenospheres as filler in aluminium casting reduce cost, decreases density and increases hardness, stiffness, wear and abrasion resistance [7]. They are considered as potential materials for components like pulleys, oil pans, intake manifolds and valve cover [8]. The use of Aluminium-fly ash composites reduces emissions like $\mathrm{CO} 2$, which otherwise come from aluminium production. In transporting the components, low weight materials consume less energy benefiting the environment [9] Zhu and Hihara [10] have reported on the corrosion performance of a continuous alumina-fibre reinforced metal-matrix composite (MMC) and its monolithic matrix alloy (Al-2\%Cu-T6) in $3.15 \mathrm{wt} \%$ sodium chloride solution.

Nunes et al [11] have studied the corrosion behaviour solution of alumina-aluminium and $\mathrm{SiC}-\mathrm{Al}$ in sodium chloride. Immersion and anodic polarisation corrosion tests have been carried out. It is reported that composites have exhibited lower corrosion resistance when compared. Nickel and copper coated reinforcements have produced beneficial effects in particular to reduction in porosity level and reduce 
interfacial reactions during the processing of metal matrix composites [12-14].

Currently, electroless deposition of metallic coatings on the ceramic reinforcement is gaining popularity owing to its advantages such as uniformity in coatings over the surfaces regardless of size and shape. Further, this technique is autocatalytic, which means no conduction surfaces are required. The published literature on advanced materials, such as Aluminium nickel-coated composites, is somewhat limited and is primarily concerned with applications of fly ash particles for the synthesis of these materials. Therefore, it was thought worthwhile to study the corrosion behaviour of this composite as well as present the pitting morphologies of the corroded surface. The present work is dedicated to such an investigation.

\section{Experimental Procedure}

A batch of $3.5 \mathrm{kgs}$ of Aluminium 6061 alloy was melted using a $6 \mathrm{KW}$ electric furnace. The melt was degassed using commercially available chlorine-based tablets (Hexachloroethane). The molten metal was agitated by use of mechanical stirrer rotating at a speed of $300 \mathrm{rpm}$ to create a fine vortex. Preheated cenospheres (preheated to $200 \mathrm{oC}$ for $2 \mathrm{hrs}$.) were added slowly into the vortex while continuing the stirring process. The stirring duration was $10 \mathrm{~min}$. The composites melt maintained at a temperature of $710^{\circ} \mathrm{C}$ was then poured into preheated metallic moulds. The stirrer blades used were made of stainless steel and were coated with a ceramic material to minimise the iron pickup by the molten metal. The amount of cenospheres was varied from 2 to $8 \mathrm{wt}$. \% in steps of $2 \%$. [15].

\subsection{Immersion corrosion test}

Immersion test were carried out as per ASTM G31 test procedure. Polished samples of Al 6061 alloy and all the composites were immersed in $3.5 \%$ $\mathrm{NaCl}$ solution for a total duration of 25 days. Weight loss measurements of the samples were done at the end of every 5 days. Corroded surfaces of the samples were cleaned with acetone before weighing using an electronic balance of accuracy of 0.001 grams. The corresponding changes in the weights were noted. The corrosion rate was calculated using the following relationship.

Corrosion rate (mpy) $\rightarrow 534 *$ weight loss / (density*area of exposure*time of exposure)

A single step activation and sensitisation step was carried out. We also used Sodium Hypophosphite to reduce Nickel in the presence of $\mathrm{PdCl} 2$. The composition of the chemicals used for the coating process is as shown in Table 1 .

$\mathrm{NiSO} 4+2 \mathrm{NaH} 2 \mathrm{PO} 2+2 \mathrm{H} 2 \mathrm{O} \rightarrow \mathrm{Ni}+2 \mathrm{NaH} 2 \mathrm{PO} 3-+\mathrm{H} 2+$ $\mathrm{H} 2 \mathrm{SO} 4$

The nickel reduction only takes place on specific catalytic surfaces, including Nickel itself, which makes the reduction process autocatalytic as shown in Equation 2. The reducing agent $(\mathrm{PdCl} 2)$ used for electroless plating not only supplies the electrons for the reduction, but some elements in the reducing agent can be incorporated into the nickel deposit improving its properties e.g. when sodium hypophosphite is used as a reducing agent the resultant deposit is a nickelphosphorus alloy.

During the plating process, the nickel sulfate and sodium hypophosphite raw materials are continuously depleted and must be replenished in order to maintain the chemical balance of the bath and addition of either ammonia or sodium hydroxide are necessary to keep the $\mathrm{pH}$ in the preferred range. This results in an accumulation of sodium and sulfate ions, along with orthophosphate, as the electroless nickel bath ages and consequently, the plating rate can decrease from $18 \mathrm{~mm} \mathrm{hr}-1$ to less than $10 \mathrm{~mm} \mathrm{hr}-1$

Table 1 Bath composition for electroless nickel deposition

\begin{tabular}{cc}
\hline Bath constituents & $\begin{array}{c}\text { Quantity } \\
\text { (g/litre) }\end{array}$ \\
\hline Nickel Chloride & 30 \\
Sodium acetate & 10 \\
Sodium Hypophosphite, & 20 \\
monohydrate (NaH2PO2, H2O) & 10 \\
Glycin (H2NCH2COOH) & 0.02 \\
\hline Sodium hydroxide &
\end{tabular}

\subsection{SEM studies}

Scanning electron microscope studies were carried out using JSM 840a Joel scanning electron microscope on Aluminium 6061, Aluminium cenosphere composite and aluminium nickel-coated cenosphere composite at Indian Institute of Science (IISc), Bangalore. The sizes of the specimens were machined to $20 \mathrm{~mm}$ (diameter)

\section{Results and Discussion}

Fig.1 to Fig. 5. shows the SEM micrographs of Nickel coated cenosphere Al 6061 composites. From the micrographs, it is observed that the Nickel coated cenosphere particles are dispersed uniformly throughout the matrix. Further, SEM images clearly indicate that 
reinforcement particulates are well bonded to the aluminium matrix and that there exist very minimum micro porosities in all the composites. This fact supports the good bond between the matrix and reinforcement. [16-17].

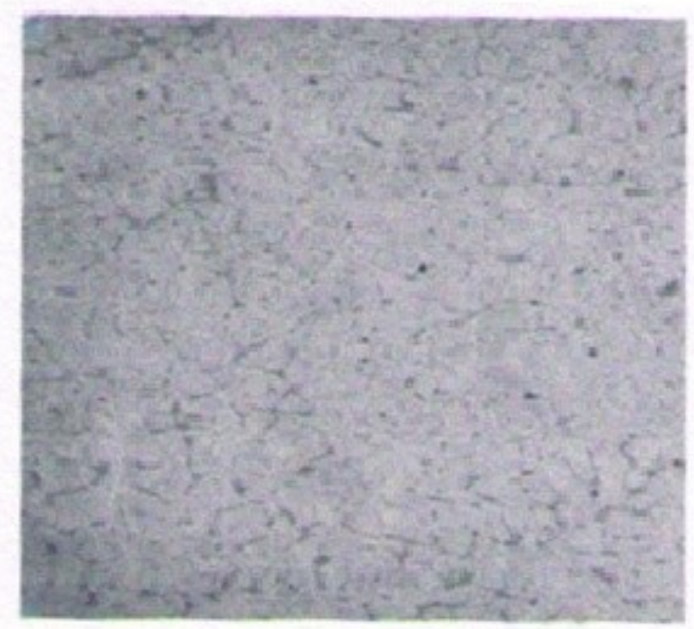

Fig. 1 Al 6061 (200x)

The few pores observed in the as-cast composite were mostly associated with the reinforcement particles and particle clusters. The latter feature was especially pronounced in the composite with a higher weight fraction of the reinforcement in the composites. The extent of distribution of reinforcement particle in the composites is more homogeneous when compared with cast composites, suggesting that rearrangement and recrystallisation had taken place.

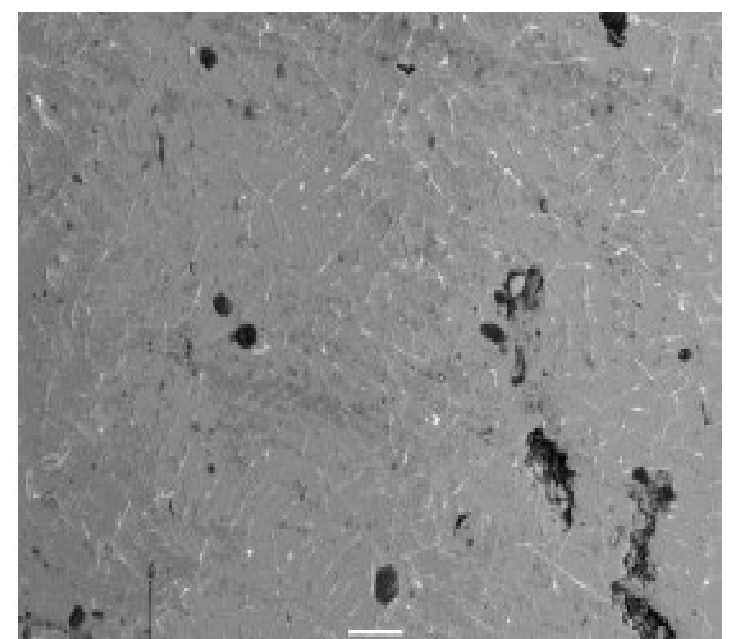

Fig. 2 Al 6061- 2\% Nickel coated cenosphere

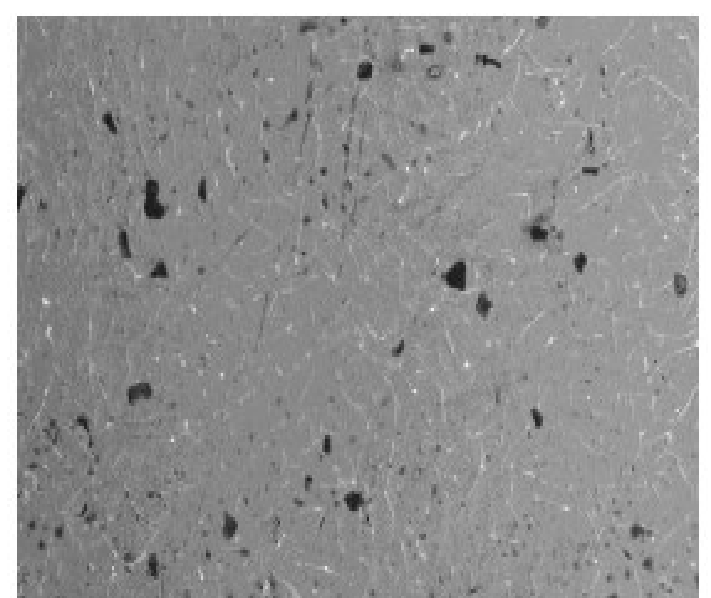

Fig. 3 Al 6061- 4\% Nickel coated cenosphere

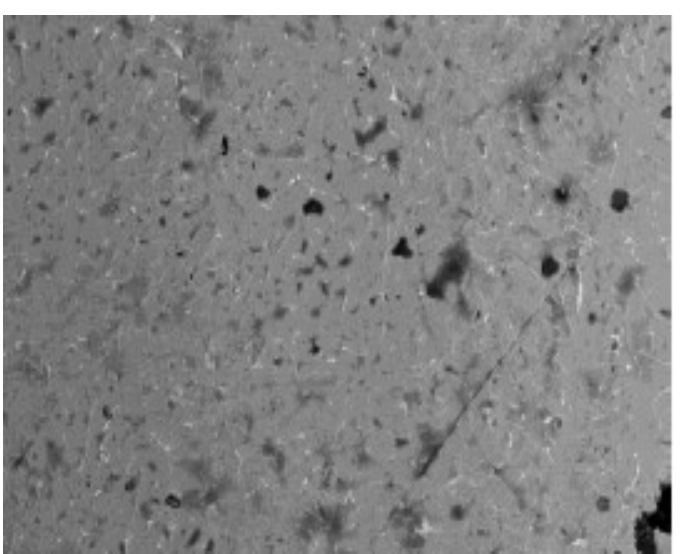

Fig. 4 Al 6061- 6\% Nickel coated cenosphere

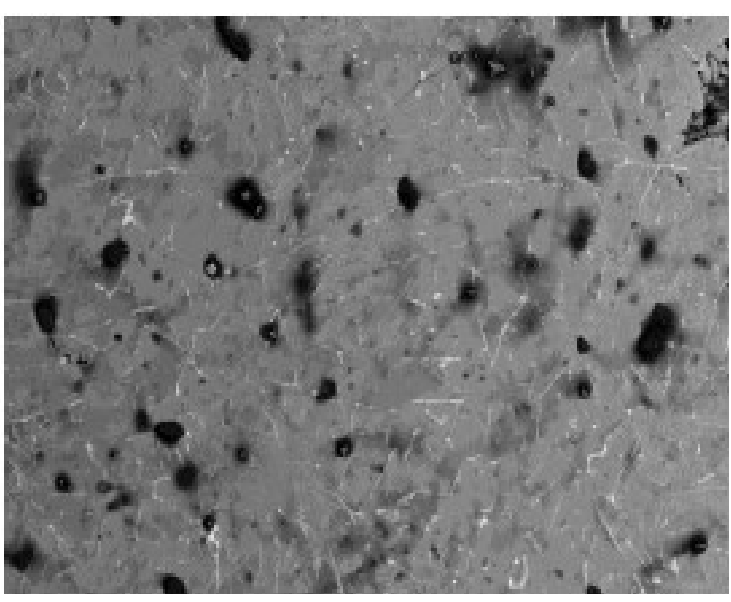

Fig. 5 Al 6061- 8\% Nickel coated cenosphere 


\subsection{Effects of Reinforcement}

Fig. 6. shows the effect of reinforcement on corrosion rate in mills per year of $\mathrm{Al} 6061$ alloy and $\mathrm{Al}$ 6061-nickel coated composites after 60 days of immersion in $3.5 \% \mathrm{NaCl}$ solution. It is observed that the corrosion rate of $\mathrm{Al} 6061$ alloy decreases with increase in the percentage of Nickel coated cenospheres as reinforcement. This can be attributed to the fact that the coating of Nickel on the cenosphere provides effective barriers for the corrosion to occur. It is therefore observed that the composite containing $8 \%$ by weight of Nickel coated cenospheres has shown better corrosion resistance when compared to its counterparts.

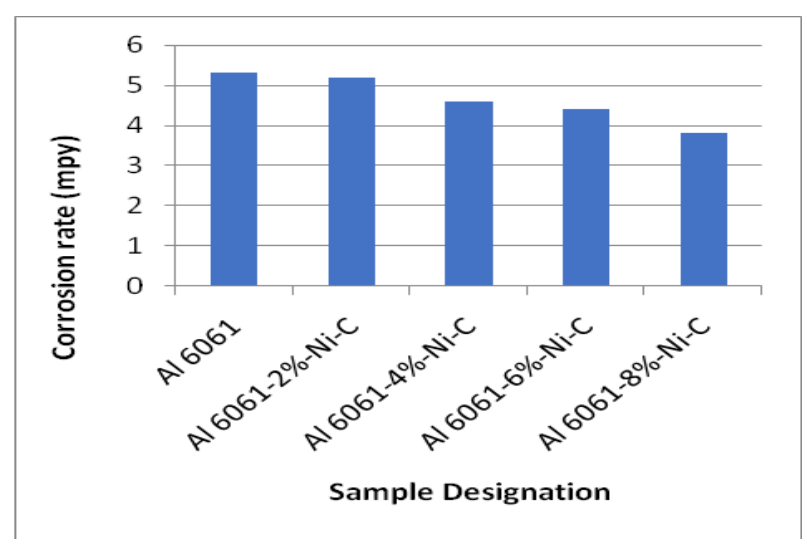

Fig. 6 Variation of corrosion rate of Al 6061 - nickelcoated composite cenospheres after 10 days of immersion in $3.5 \% \mathrm{NaCl}$ solution

\subsection{Effect of immersion duration}

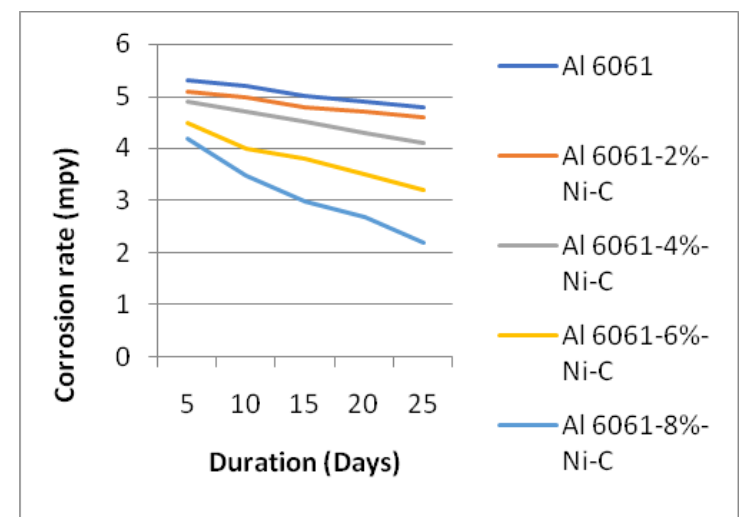

Fig. 7 Variation of corrosion rate in mpy of Al 6061 matrix alloy and Al 6061-nickelcoated cenosphere composites
Fig. 7 shows the corrosion rate in mpy as a function of immersion time in the number of days in $3.5 \% \mathrm{NaCl}$ for $\mathrm{Al} 6061$ matrix alloy and Nickel coated cenosphere composites. It is observed that the corrosion rate drops drastically in the composites studied. It is further noted that the corrosion rate of $\mathrm{Al} 6061$ matrix alloy and its composite systems varies in a narrow band. This can be attributed to the facts as discussed in the earlier section

\subsection{Pitting morphology}

Fig. 8 and Fig. 9 shows the SEM photographs of the corroded surface of Al 6061 matrix alloy and Al 6061-nickel coated cenosphere composites, respectively. It is observed that the developed composites possess a lesser number of pits when compared with the $\mathrm{Al} 6061$ matrix alloy. Bhat et al. [18] have observed a similar trend in his study on aluminium matrix composites

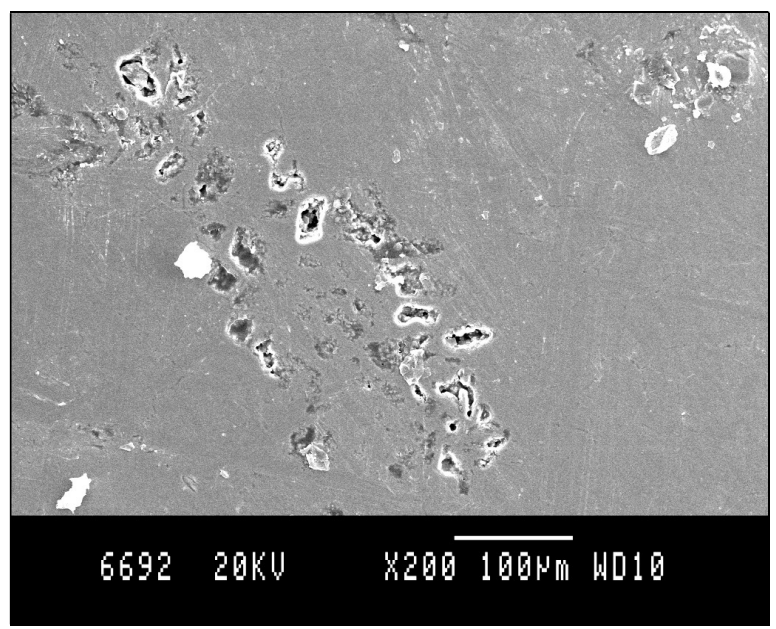

Fig. 8 Al 6061 alloy (200x)

Fig. 10 shows the SEM photographs of the corroded surface of Al 6061-8 wt\% Nickel coated cenosphere composites for varying time durations. It is observed that the damage on the composite decreases with an increase in the percentage of reinforcement. However, increase in time duration has led to the formation of a passive layer on to the surface of the composite material leading to decrease in the corrosion rate days which is well supports the obtained results discussed in earlier section. 
Journal of Manufacturing Engineering, September 2020, Vol. 15, Issue. 3, pp 054-059

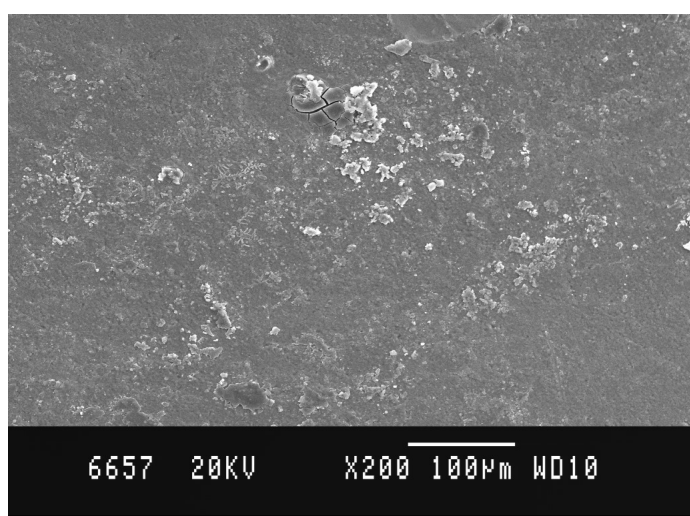

Fig. 9(a) Al 6061-4wt\% nickel coated cenosphere $(200 x)$

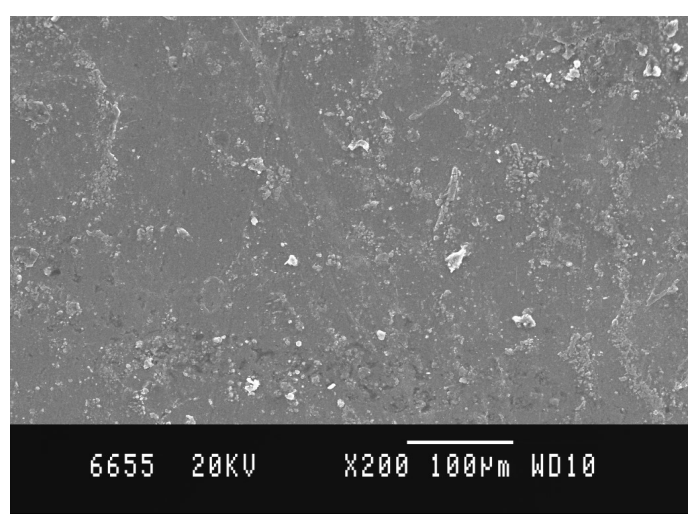

Fig. 9(b) Al 6061-8 wt\% nickel coated cenosphere $(200 x)$

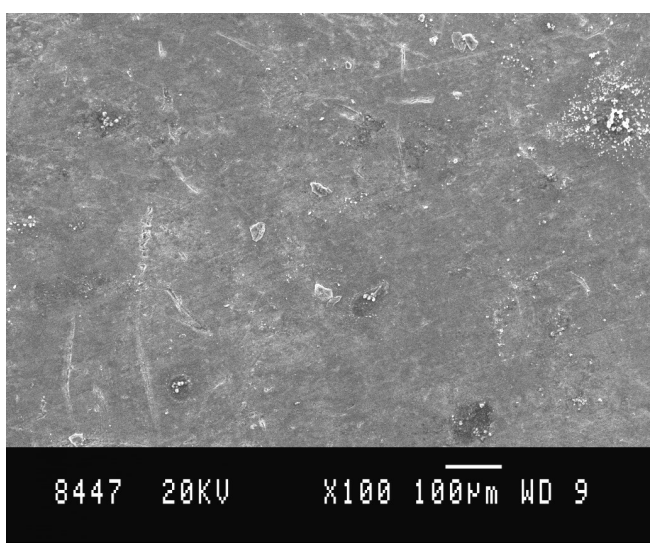

Fig. 10(a) 5 days (200x)

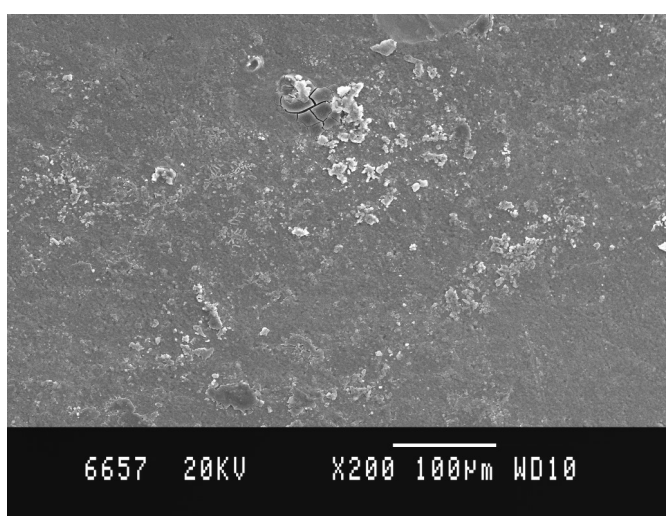

Fig. 10(b) 15 days (200x)

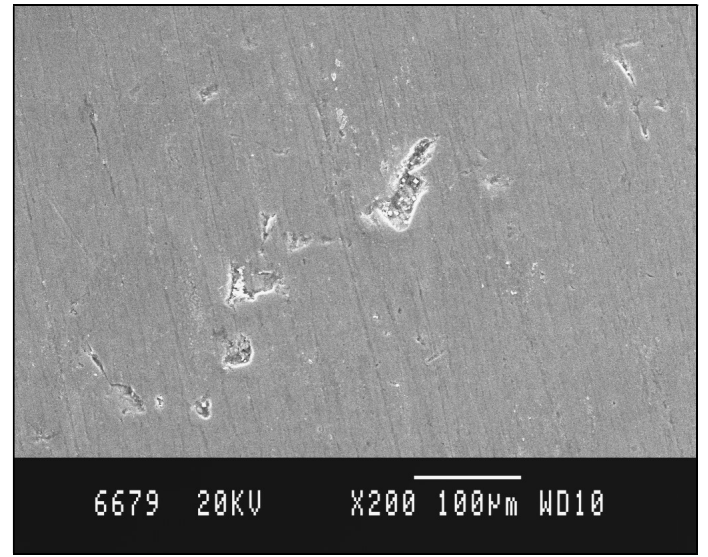

Fig. 10(c) 20 days $(200 x)$

Fig. 10 SEM photographs of corroded surfaces of Al 6061-8 2wt\%nickel coated cenosphere composites for different time durations

\section{Conclusions}

i. Al 6061-nickel coated cenosphere composites have been successfully produced by liquid metallurgy route.

ii. Al 6061-nickel cenosphere composites (8\%) possess inferior corrosion resistance in $3.5 \% \mathrm{NaCl}$ medium when compared with Al 6061 alloy.

iii. It is observed from the pitting morphology studies that the pits are deeper in the reinforced composites as compared to the unreinforced counterparts.

iv. The studies clearly show that the reinforcement has, in particular, a higher role to play in the improvement of corrosion resistance of the composite. 


\section{References}

1. TanusreeBera and Samir K Acharyam (2018), Study of the tribological properties of fly ash cenosphere particulate reinforced LM6 (Al-Si12) matrix alloy composites produced by stir casting method, Tribology, 18:124-135.

2. Ahmed A and Prashanth T (2018), Mechanical Property Evaluation Aluminium 6061 Nickel Coated Cenosphere Composites, Mechanics and Mechanical Engineering, 22. $1381-1388$.

3. Shankar, $S$ and Harichandran $S$ (2019), Aluminium alloy reinforced with fly ash: Techniques and particle size, JurnalTribologi, 23:13-37

4. Anand Kumar, Seetharamu S (2019), Jaganath Nayak and Satapathy L N, A Study on Thermal Behavior of AluminumCenosphere Powder Metallurgy Composites Sintered in Microwave, Procedia Materials Science, 5:1066-1074.

5. Guo $R Q$, Venugopalan $D$ and Rohatgi $P K$ (1998) Differential thermal analysis to establish the stability of aluminum-fly ash composites during synthesis and reheating, Materials Science and Engineering: A, 241 184-190.

6. Ravi Kumar V, Suresh R, Prakash Rao C R, Ravi Kumar D $V$ and Bharat $V$ (2018), Effect of Heat Treatment on Tensile and Corrosion Properties of LM6 Hybrid Metal Tensile and Corrosion Properties of LM6 Hybrid Metal
Matrix Composite Reinforced with Cenosphere and Red Mud, Journal of Minerals and Materials Characterization and Engineering, 7:1-17.

7. Sivakumar $K N$, Argade $S D$, Sadler $R L$, Sharpe $M M$ Dunn $L$ and Swaminathan $G$ (2006), processing and properties of a lightweight fire resistant core material for sandwich structures, Journal of Advanced Materials, 38 . $32-38$.

8. Mishra S R, Kumarb S, Waghc A, Rhoc $J Y$ and Gheyid T (2003), Temperature-dependent surface topography analysis of Illinois class F flyash using ESEM and AFM, Materials Letters, 57:2417-2424.
9. Saravanan Varatharaju $P R$, Thyla $S R$, Balakrishnan (2015), An Experimental investigation on Properties of Cenosphere reinforced Aluminium metal matrix composite", International Journal of Innovative Research in Science, Engineering and Technology, 4:10399-10407

10. Metzoer M and Fishman $S$ G (1983), Corrosion of Metal Matrix Composites, Ind. Engg. - Chem. Prod. Res. Dev., 22:296-302

11. Paciej and Agarwala (1988), Influence of processing variables on the corrosion susceptibility of metal-matrix composites, corrosion, 44 (10):680-684.

12. Chang $S V$ and Lin $S J$ (1996), Fabrication of SiCp reinforced copper matrix composites by electroless copper plating, Scientific Materials, 35:225-231.

13. Ramesh C S, Anwar Khan A $R$ and Safiulla $M$ (2003), Wear and Corrosion Behaviour of Al6061-TiO2 Composites, Material Science Forum, 437-438:317 - 320,

14. Taneoka, Odawara O, and Kaieda Y (1989), Combustion synthesis of the titanium-aluminum-boron system, Journal of American Ceramic Society, 72, 1047-1049.

15. Warrier and Lim (1993), Silver coating on carbon and silicon carbide fibres, Journal of Material Science, 28: 4868-4877.

16. Axen N, Alahelisten A and Jacobson S (1994), Abrasive wear of alumina fibre-reinforced aluminium, Wear, 17: wear of ah 104.

17. Mahendra $K V$ and Radhakrishna $K$ (2007), Effect of particle size on mechanical properties and tribological behavior of aluminium fly ash composites, Mat. Sc.-Poland, 25:57-68

18. Bhat $M S N$, Surappa $M K$ and Naik $H V S$ (2001), Corrosion behaviour of $\mathrm{SiC}$ particle reinforced 6061/Al alloy composites, Journal of Material Science, 26 (18): 49-91. 